\title{
Single center experience of hepatic artery reconstruction during living donor liver transplantation: microscope versus surgical loupe
}

Changho Seo

Division of Hepatobiliary, Department of Surgery, Seoul St. Mary's Hospital, College of Medicine, The Catholic University of Korea, Seoul, Korea

Background: Hepatic artery (HA) reconstruction during living donor liver transplantation (LDLT) is the key step due to the small diameter of the artery and risk of HA thrombosis (HAT). To overcome this risky procedure, it has been preferred to using microscope during HA reconstruction by experienced microsurgeon. However, it takes long time to complete the procedure and has long and steep learning curve. To make this procedure simple, some transplant surgeons recently try the procedure using surgical loupe. We conduct this study to compare the outcomes after HA reconstruction using conventional microscope versus surgical loupe.

Methods: We retrospectively reviewed outcomes of 300 LDLTs at Seoul St. Mary's Hospital from April 2014 to July 2020. From April 2014 to September 2017 (era 1), HA reconstruction was performed with conventional microscope by an experienced plastic surgeon. From September 2017 to end date (era 2), it was performed with surgical loupe $(\times 5.0)$ by an experienced transplantation surgeon.

Results: There was no difference in most perioperative outcomes between two groups including major postoperative complications: HAT (2/150 vs. $1 / 150, P=0.562)$, postoperative bleeding (13/150 vs. $6 / 150, P=0.097)$, and biliary leak (18/150 vs. $13 / 150$, $P=0.343)$. It was statistically significant between two groups for total operation time $(436.66 \pm 83.91$ minutes vs. $415.35 \pm 68.55$ minutes, $P=0.035$ ). Multivariable regression modeling to adjust for baseline differences showed that the use of surgical loupe was not associated with HA thrombosis.

Conclusions: HA reconstruction with surgical loupe makes results as good as with microscope for the transplant surgeon and contributes to reducing operating time.

Corresponding author: Changho Seo

E-mail: schjee17@gmail.com

\section{(c) The Korean Society for Transplantation}

This is an Open Access article distributed under the terms of the Creative Commons Attribution Non-Commercial License (http://creativecommons.org/licenses/by-nc/4.0/) which permits unrestricted non-commercial use, distribution, and reproduction in any medium, provided the original work is properly cited. 